Nouvelles perspectives en sciences sociales

\title{
La systémique, penser et agir dans la complexité, de Gérard Donnadieu et Michel Karsky (2002), Éditions Liaisons, Paris.
}

\section{Rachid Bagaoui}

Volume 1, numéro 2, mars 2006

URI : https://id.erudit.org/iderudit/602474ar

DOI : https://doi.org/10.7202/602474ar

Aller au sommaire du numéro

Éditeur(s)

Prise de parole

ISSN

1712-8307 (imprimé)

1918-7475 (numérique)

Découvrir la revue

Citer ce compte rendu

Bagaoui, R. (2006). Compte rendu de [La systémique, penser et agir dans la complexité, de Gérard Donnadieu et Michel Karsky (2002), Éditions Liaisons, Paris.] Nouvelles perspectives en sciences sociales, 1(2), 219-224.

https://doi.org/10.7202/602474ar d'utilisation que vous pouvez consulter en ligne. 


\title{
Compte rendu de l'ouvrage La systémique, penser et agir dans la complexité De Gérard Donnadieu et Michel Karsky (2002) Éditions Liaisons, Paris
}

\author{
RACHID BAGAOUI \\ Université Laurentienne \\ Sudbury, Ontario, Canada
}

La systémique, penser et agir dans la complexité est un livre stimulant à plus d'un égard. Il nous présente avec conviction les acquis de la systémique, comme expression de la pensée complexe, qui se veut, selon les souhaits des auteurs, un guide qui permettrait aux chercheurs et aux décideurs de s'orienter dans notre monde de plus en plus complexe. Le lecteur désireux d'en savoir plus sur la pensée complexe est bien servi par le livre de Donnadieu et Karsky. La pensée systémique est exposée dans ses détails. Destinés aux hommes et aux femmes en position de responsabilité (décideurs, politiciens, dirigeants d'entreprises...). Le livre nous fait bien comprendre que les phénomènes non seulement sont complexes mais qu'il faut une approche complexe pour les appréhender. Et la pensée systémique remplit cet objectif. Dans les systèmes complexes, contrairement aux systèmes simples, interviennent plusieurs variables dont l'effet demeure insaisissable. Les systèmes complexes se caractérisent aussi par un nombre élevé de composantes. En matière de prise de décision dans les systèmes complexes, il y a une diffusion des centres de décision et de l'autorité. Enfin, la décomposition entre composantes est faible. 
Bien que son application soit parfois difficile, la pensée complexe offre une base pour comprendre les objets complexes. Une entreprise, suivant la perspective des auteurs, est un exemple de cette réalité complexe, car le milieu dans lequel elle évolue - par exemple le marché, le cadre juridique, les technologies, la concurrence, les échanges, les relations sociales - se présente plutôt comme une situation complexe. Le chercheur ou le praticien qui s'attaque à cet objet et à cette situation complexe est forcément confronté à quatre aspects de la réalité : l'imprécision (on ne peut dire avec quelle précision quelles sont les dimensions et les frontières du système et de ses composantes.) ; l'instabilité (le temps agit sur les systèmes complexes en les désorganisant et en faisant émerger l'ordre du désordre ou, si l'on veut, l'autoorganisation) ; l'ambiguité (un objet complexe entretient tellement d'interactions avec son environnement qu'il se révèle en fin de compte incompréhensible et incontrôlable) ; l'incertitude (les systèmes complexes arrivent à développer des comportements libres et autonomes par rapport aux déterminismes extérieurs).

Les objets complexes possèdent de nombreuses propriétés que l'on peut désigner par le terme Systèmes Hyper Complexes (SHC). Les SHC sont à la fois clos et ouverts ; ils sont définis par leurs relations (le tout est plus que la somme des parties) ; ils sont hiérarchisés en niveaux d'organisation (à chaque niveau émerge des propriétés nouvelles et spécifiques) ; ils sont finalisés (exister pour un but donné) ; ils ont besoin de variété ; ils sont autoorganisateurs (capacité de changer, tout en restant le même).

L'approche systémique est une manière particulière d'aborder un objet complexe comme celui de l'entreprise. Née aux États-Unis au début des années cinquante, connue et adoptée en France depuis les années soixantedix ${ }^{1}$, elle repose sur les postulats suivants :

- un système a une limite qui le sépare de l'environnement;

- un système assemble des parties, ou des éléments, ou des composants de nature différente ;

- il existe entre les éléments des relations de diverses natures qui se manifestent le plus souvent sous forme d'un réseau de communication permettant l'échange entre les principaux composants du système ;

\footnotetext{
${ }^{1}$ En fait, pour comprendre la pensée systémique il faut remonter beaucoup plus avant dans l'histoire des sciences. Voir à ce sujet la contribution de Gérard Donnadieu, « Systémique et science des systèmes. Quelques repères historiques 》 (fait, à Ville D'avray, le 8 mars 2004), 24 p. Dans : Site consulté en octobre 2005 : www.afscet.asso.fr/HistoireSystemique.pdf.
} 
- dans ce réseau, certaines des parties ou certains des composants jouent un rôle de transformation des produits échangés ;

- d'autres composants jouent enfin le rôle de vannes et servent à contrôler les échanges; ils sont les organes de base de la régulation et agissent selon le mécanisme de la rétroaction;

- tous ces éléments sont agencés pour atteindre un ou plusieurs objectifs communs.

L'approche systémique repose aussi sur un certain nombre de concepts comme la rétroaction (feed-back); la régulation des systèmes; les boucles positives, négatives et ago-antagonistes. Mais, comme le disent les auteurs :

Toutes définitions de la notion de système, au-delà de leur intérêt intellectuel et de leur utilité pour délimiter le champ d'observation, sont peu opératoires pour l'action. La richesse du concept de système ne se dévoile en effet que par l'utilisation qui en est faite. La seconde partie du présent ouvrage, plus particulièrement la partie relative aux applications, pourra seule en montrer la fécondité.

C'est pourquoi, dans le chapitre 3, les auteurs exposent la méthode systémique qui repose sur une relation dialectique entre le sujet et l'objet. Le sujet projette sa représentation sur l'objet qui induit une représentation sur le sujet. Notre intelligence construit des représentations des objets du monde extérieur (sous forme d'idées, de concepts, de cartes, de schémas...) qui nous permettent de nous orienter dans ce monde et d'agir sur lui. Pour ce faire, l'analyse des systèmes consiste :

- à définir les limites du système, à modéliser, à identifier les éléments importants et les types d'interactions entre ces éléments, puis à déterminer les liaisons, à dégager et à identifier les variables de flux et d'état, les boucles de rétroaction, les délais ;

- à situer le système dans son environnement et à comprendre la nature des échanges entre le système et son environnement, à avoir une idée de son architecture interne, et à connaitre l'histoire du système pour comprendre son évolution. L'outil utilisé pour amorcer une telle exploration est la triangulation systémique composée d'un aspect fonctionnel (ce que le système fait), d'un aspect structural (comment le système est composé) et d'un aspect historique (ce que devient le système). Afin de mettre en œuvre de manière méthodique le volet structural de la triangulation, on utilise le découpage systémique qui s'appuie sur certains critères (finalité, historique, niveau d'organisation, structure) ; 


\section{2 NPSS, VOLUME 1, NUMÉRO 2, 2006}

- à construire un modèle (modélisation) à partir des données de l'analyse de systèmes ; on s'efforce d'y faire apparaitre les différents flux ainsi que les actions de pilotage sous-jacentes à la régulation du système; l'analyse peut, par son caractère dynamique, déboucher sur des modèles analogiques.

L'approche systémique repose sur plusieurs processus et étapes :

- l'analyse causale (modélisation qualitative) : Comment fait-on ? Quelles variables prendre en considération ? Par où commencer? Y a-t-il des variables plus significatives que d'autres?

- la modélisation : Définir un schéma causal, choisir les variables qui paraissent être des variables d'accumulation (variables d'état). Une fois ces variables choisies, il faut se poser la question : quels sont les flux qui remplissent ou qui vident ces variables de niveau? Ensuite, il faut se demander de quoi dépendent ces flux, c'est-à-dire de quelles variables d'information ou de décision, de quelles influences, de quelles contraintes?

- la formalisation et la quantification : formaliser les relations, quantifier et paramétrer.

- la simulation : Tester le modèle, en introduisant enfin explicitement la variable temps. Analyser les dynamiques possibles, mettre en évidence des comportements imprévus, réaliser et étudier des scénarios qui seront toujours moins coûteux que des essais réels. Faire de la prospective, modifier le modèle mental, s'attaquer éventuellement à la phase ultime, à savoir les modifications de structure destinées à générer les comportements désirés (modification de la réalité).

- le calibrage et la validation : Vérification expérimentale, confrontation avec des statistiques existantes et réglage du modèle pour tenter de minimiser les différences entre résultats de simulation et séries historiques. $\dot{A}$ ce niveau interviennent les avis d'experts, puis la simulation partielle ou totale du modèle.

Pour sortir de l'abstraction et de l'aridité de l'exposé, les auteurs nous présentent dans la seconde partie plusieurs études correspondant à des applications réalisées par les auteurs de ce livre sur les champs les plus divers. En voici deux exemples : 
L'étude sur un rite initiatique sert à illustrer la mise en œuvre de la triangulation systémique. Elle repose sur l'aspect fonctionnel : on cherche à répondre à la question à quoi sert le rite ? sur l'aspect structural : on cherche à décrire la structure interne du rite, les relations, les correspondances, les analogies entre ses divers composants mythicorituels; sur l'aspect historique : on cherche à comprendre les lois de développement du rite.

L'étude d'une organisation illustre l'application de l'aspect structural. Il s'agit d'identifier l'environnement technico-économique, l'origine et le débouché des flux d'exploitation, le réservoir de ressources économiques, financières, technologiques. Il s'agit aussi de décrire l'environnement organisationnel qui comprend les différents pouvoirs politiques, réglementaires. Ces différents pouvoirs s'expriment sous forme de lois, de règlements ou de normes. Ces formes de régulations sociales ont pour fonction de limiter l'autonomie de décision du système organisationnel. L'environnement social renvoie également aux multiples appartenances du personnel au sein de l'organisation dans laquelle il vit (famille, syndicat, parti politique, église, association...). Cet environnement est soumis aux grands changements culturels que connaissent nos sociétés. Enfin, dans une organisation, il y a un système de pilotage des informations en vue de produire des décisions qui se traduiront par des actions.

Les auteurs donnent d'autres exemples pour illustrer l'approche systémique : celui d'un chantier de forage d'exploration; celui d'une analyse du fonctionnement et des prospective de l'un des principaux marchés de matières premières. Pour terminer leur livre, ils poussent à son point ultime la modélisation ; doté d'un formalisme mathématique et de relations quantifiées, le modèle devient opérable sur ordinateur et se prête ainsi à la simulation dynamique.

En conclusion, je dois souligner que ce n'est pas un hasard si j'ai insisté tout au long de mon résumé sur la pensée systémique : l'objet du livre n'est pas l'étude des objets complexes en eux-mêmes mais bel et bien la pensée systémique. Comme je l'ai indiqué en introduction, bien que l'application de la systémique soit parfois difficile, elle offre une base pour comprendre les objets complexes, les décrire dans leur globalité, en essayant d'en appréhender, à la fois, la finalité, les éléments, les relations entre ces éléments et les mécanismes qui en assurent l'évolution. J'aimerais terminer mon résumé sur un commentaire. Je trouve que les auteurs n'ont pas manifesté de la distance ni un esprit critique, condition de tout travail 


\section{NPSS, VOLUME 1, NUMÉR0 2, 2006}

intellectuel, face à leur propre approche (la complexité en général et la systémique en particulier). J'aurais aimé que les auteurs discutent les limites des ces approches, les objets qui peuvent échapper au regard du systémique, etc. En ne soumettant pas la théorie à la critique, en abdiquant la distance, les auteurs reprennent à leur compte non seulement la théorie mais le discours sur la théorie et les présupposés derrière ce discours. Je suis tenté de dire que le livre est une contribution à la production de la croyance dont il fallait décrire les fondements et les effets sociaux. Peutêtre qu'une section à la fin du livre aurait réglé ce problème.

Cela étant dit, les décideurs trouveront sûrement dans l'approche présentée les éléments de base pour comprendre et affronter la complexité du monde qui les entourent. 